\title{
NBO Analysis Based on CPLD Between Water Molecules Hydrogen Bonds
}

\author{
Ziwei Li \\ North China Electric Power University, Baoding ,China \\ 1533191873@qq.com
}

Keywords: water molecules, hydrogen bonds, Gaussian, NBO, CPMD

\begin{abstract}
By using Gaussian software to make input file of water molecules, then in the Linux environment, the parallel CPMD was applied to simulate the nature of the hydrogen bond in aqueous solution through trajectory computation of input file. Then extract the track fragments selectively which meets hydrogen bonds formation conditions and switch to NBO analysis of trajectory segments under Linux. Finally drawing the acquisition after the analysis of data collection can intuitively see changes of the relations between various data during the process of the hydrogen bond formation and fracture.
\end{abstract}

\section{Introduction}

Water is very common in our life, but it has some inexplicable properties such as the maximum density $(1 \mathrm{~g} / \mathrm{cm} 3)$ under an atmospheric pressure $(105 \mathrm{pa})$, temperature of $4{ }^{\circ} \mathrm{Cand}$ freezing at $0{ }^{\circ} \mathrm{C}$, boiling in $100{ }^{\circ} \mathrm{C}$. These phenomena are very common but it is hard to explain, and now an explanation is recognized because of hydrogen bonding between water molecules and produce these phenomena.In this paper, trajectory calculation of water molecules group model established by Gaussian is presented using CPMD and more appropriate bonding trajectory is also chosen to analysis hydrogen bonds atomic spacing before and after, E (2) (secondary order perturbation stabilization energy), bond angles changes and the relationship between the number of occupied electron and strength of hydrogen bonds ${ }^{[1]}$.

\section{The experimental method}

\subsection{First-Principles}

First Principles ${ }^{[2]}$ of quantum mechanics calculation refers to only the five basic physical constants $(\mathrm{m} 0, \mathrm{e}, \mathrm{c}, \mathrm{h}, \mathrm{kB})$ included and does not depending on any empirical parameters can the the state and properties of micro system reasonably predicted .

First-principle calculation method has incomparable advantages compared with semi-empirical method, because it only needs to know the atomic number of each element constituting the microscopic system, without the acquisition for any other adjustable (experience and the fitting) parameters and apply quantum mechanics to calculate the physical properties, total energy of the micro system, electronic structure, etc.

On the one hand, first principles calculation is a supplement besides of true experiment, because the characteristics and properties of simulation system can be more close to the real situation by calculating. On the other hand, compared to the real experiment, first-principles calculations allow us to quickly design the experiment to meet the requirements.

\subsection{Operation method}

The CPMD software is a program processed under the Linux environment,serving computation of the model which we want to analysis by using the First-principle .

The model of the three water molecules(showed as Fig 1) is established by Gauss-view in this paper and dealt with in an empty cell in order to satisfy the boundary conditions to simulate the aqueous environment. 


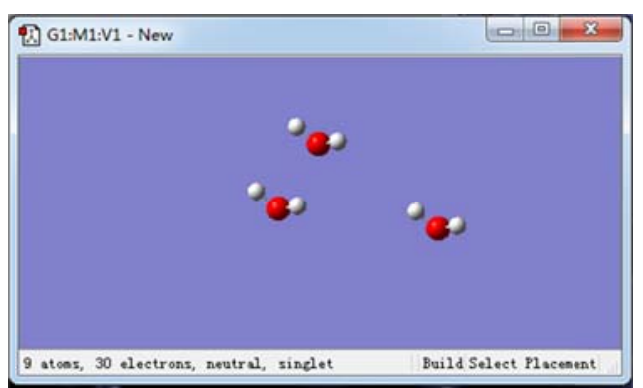

Fig 1 Initial water molecules model under the Gaussian

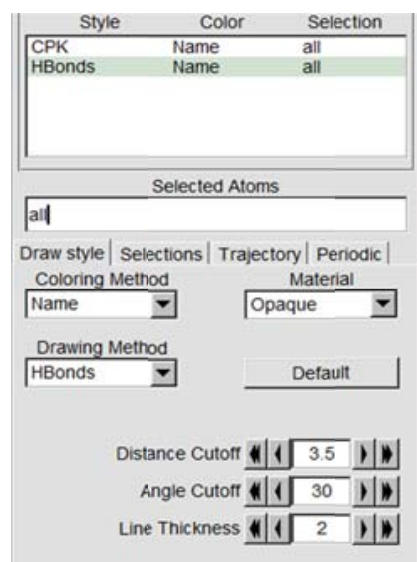

Fig 2 Parameters determination

Then put the completion of the output .gif file under the Linux environment ,calculated through parallel CPMD, setting 120000 steps simulation and output the analog track file, using VMD open trajectory file to set bonding parameters.

Set the parameters as follows:

Play the trajectory to find the right key into the track and make trace captured, and statistic the track between 39650--82444 steps to find the suitable track.It is found that the formation and fracture of hydrogen bonds between 65285-65285 steps is more suitable for study, so trajectory files on 64552-65285 track segments are sampled and recorded every five steps (Owing to the large manual sampling, making use of VB programming to realize automatic sampling).The final sample obtained 147 track files, each file is amended to Gaussian G03 input file under Linux,and calculated gradually(Using shell scripts to calculate the 147 file automatically and put each of the output file inputted itemized saved).

Next, keep the statistics of data change of the distance between the atoms related to hydrogen bonds in the track file and plotted, then count the $\mathrm{E}(2)$ second-order perturbation stabilization energy associated with each step of hydrogen from each NBO output file and mapping, then statistics the occupation numbers of the related electrons and atoms before and after atomic hydrogen bonding ${ }^{[3]}$.

\section{Result and analysis}

Each atoms related to the hydrogen bonds have been marked in order to facilitate the research,which showed as Fig 3.

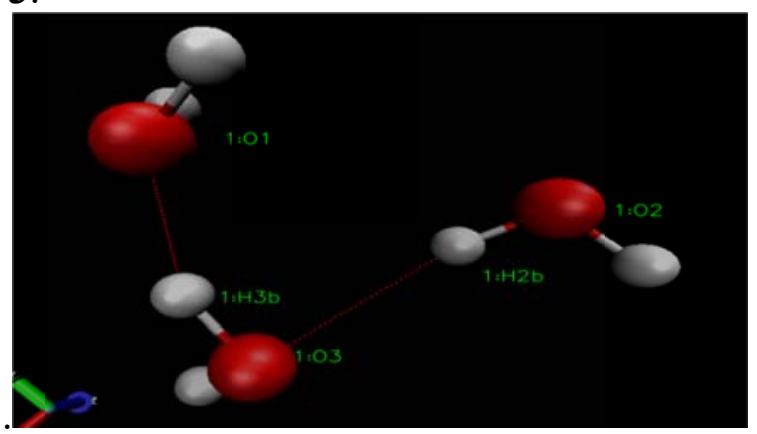

Fig 3 Track sample

The $\mathrm{O} 3-\mathrm{H} 3 \mathrm{~b} \cdots \mathrm{O} 1$ in the beginning of the sample trajectory meets the hydrogen bond forming conditions given early. Then the space between the bonding atoms and the $\mathrm{E}(2)$ were observed when water molecules moving in freedom ${ }^{[4]}$.

According to the observation, a phenomenon that three water molecules just form two Hydrogen Bonds(The $\mathrm{O} 2-\mathrm{H} 2 \mathrm{~B} \cdots \mathrm{O} 3$ is emerged) in the 50 step was found .But it can be clearly discovered that four groups of atomic spacing are always in the bonding range of hydrogen bond from the Fig 4. 
Switching to the Fig 5, we could find the E(2) in the O2-H2B $\cdots \mathrm{O} 3$ reached $3.36 \mathrm{kcal} / \mathrm{mol}$ which is higher than the previous at 50st step, the bond angle up to the critical value 120 。 and the electron occupation number of $\mathrm{O} 3$ also ascend to the highest level 0.00579 at the same time.(The black represents the $\mathrm{O} 3-\mathrm{H} 3 \mathrm{~B} \ldots \mathrm{O} 1$ and the red represents the $\mathrm{O} 2-\mathrm{H} 2 \mathrm{~B} \ldots \mathrm{O} 3$ )
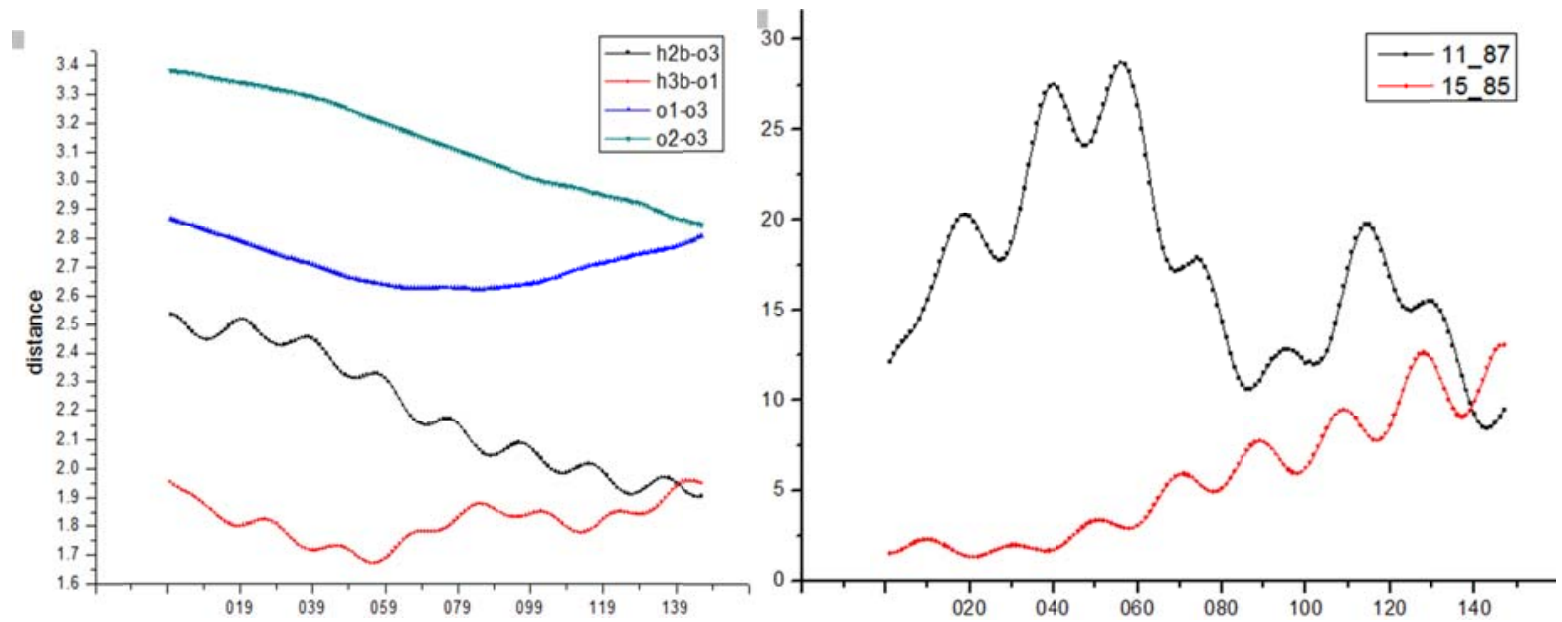

Fig 4 Atomic spacing changes

Fig 5 Hydrogen bonds between atoms of E (2)

On the whole,there is showing a rising trend in the following sections except for some fluctuations and distance between atoms becomes small as well as the angle of hydrogen bond get big, which shows hydrogen bond becoming more stable .

On the contrary, atomic spacing of the $\mathrm{O} 3-\mathrm{H} 3 \mathrm{~b} \ldots \mathrm{O} 1$ is always small and the $\mathrm{E}(2)$ very high .However,the $\mathrm{E}(2)$ plummeted after the $58 \mathrm{st}$ step as is reduced to $20.71 \mathrm{kcal} / \mathrm{mol}$ at the $64 \mathrm{st}$ step.Moreover , the electron occupation number of $\mathrm{O} 1$ has been broken down to 0.002165 at the 64st step and hydrogen bond broke, but the $E(2)$ is still much bigger than the $E(2)$ while the the $\mathrm{O} 2-\mathrm{H} 2 \mathrm{~B} \ldots \mathrm{O} 3$ come into being. The bond angle has been less than the minimum critical angle 120 。 right now.

Thus it can be seen that bond angle is so small that cannot form a hydrogen bond though the $\mathrm{E}(2)$ has a very large amount .

Continuing to observe the situation, the $\mathrm{O} 3-\mathrm{H} 3 \mathrm{~B} \cdots \mathrm{O} 1$ of hydrogen bond formed again at the $117 \mathrm{st}$ step when the $\mathrm{E}(2)$ peaked to $19.00 \mathrm{kcal} / \mathrm{mol}$ after the decay .Observing the bond angle of the NBO output and it is just reaching a critical point .

\section{Conclusion}

Based on the analysis of the above, it can be concluded that the $\mathrm{E}(2)$ is not the decisive factors of hydrogen bond formation but the key factor lies in the bonding point of view. The $\mathrm{O}$ to $\mathrm{H}$ attractions couldn't form a stable state without a right angle. Simultaneously, each atom's electron occupation number and $\mathrm{E}(2)$ have similar trends before and after the formation of hydrogen bonds. The two elements determine the degree of stability of hydrogen bond .

\section{References}

[1]Shuwei Xia, Xiang Xu, Hong Yu, Huiling Zhang. Acrylonitrile on $\mathrm{Cu}$ (111) surface chemical adsorption density functional research and NBO analysis.Chemical Journal of Chinese Universities.2007,04:751-754.

[2]Guoxun Wu.Based on first principles of dislocation motion characteristics of the molecular simulations.Harbin Institute of Technology,2008.0484.1

[3]Xia Zhang, Qiang Zhang, Dongxia Zhao.The definition of aqueous solution of hydrogen bond life and relaxation mechanism.Acta Physico-Chimica Sinica. 2011,11:2547-2552.ISSN:1000-6818 
[4]Yongping Zeng, Xiaomin Zhu, Zhanghua Yang.Water, methanol and ethanol liquid Car Parrinello microstructure properties of molecular dynamics simulation.Acta Physico-Chimica Sinica.2011,12:2779-2785.ISSN:1000-6818 